\title{
Some properties of generalized Fisher information in the context of nonextensive thermostatistics
}

\author{
J.-F. Bercher \\ Laboratoire d'informatique Gaspard Monge, UMR 8049 \\ ESIEE-Paris, Université Paris-Est \\ 5 bd Descartes, 77454 Marne la Vallée Cedex 2, France \\ tel: 33-1-45-92-65-15 fax: 33-1-45-92-66-99
}

\begin{abstract}
We present two extended forms of Fisher information that fit well in the context of nonextensive thermostatistics. We show that there exists an interplay between these generalized Fisher information, the generalized $q$-Gaussian distributions and the $q$-entropies. The minimum of the generalized Fisher information among distributions with a fixed moment, or with a fixed $q$ entropy is attained, in both cases, by a generalized $q$-Gaussian distribution. This complements the fact that the $q$-Gaussians maximize the $q$-entropies subject to a moment constraint, and yields new variational characterizations of the generalized $q$-Gaussians. We show that the generalized Fisher information naturally pop up in the expression of the time derivative of the $q$ entropies, for distributions satisfying a certain nonlinear heat equation. This result includes as a particular case the classical de Bruijn identity. Then we study further properties of the generalized Fisher information and of their minimization. We show that, though non additive, the generalized Fisher information of a combined system is upper bounded. In the case of mixing, we show that the generalized Fisher information is convex for $q \geq 1$. Finally, we show that the minimization of the generalized Fisher information subject to moment constraints satisfies a Legendre structure analog to the Legendre structure of thermodynamics.
\end{abstract}

Keywords: Generalized Fisher information, Generalized Rényi and Tsallis

Email address: jf.bercher@esiee.fr (J.-F. Bercher)

This is a preprint version that differs from the published version, Physica A, vol. 392, issue. 15, pp. 3140-3154, doi:10.1016/j.physa.2013.03.062, in some improvements after refereeing, pagination and typographics details. 
entropies, Generalized q-gaussian distributions, Information theoretic inequalities

PACS: 02.50.-r, 05.90. $+\mathrm{m}, 89.70 .+\mathrm{c}$

\section{Introduction and preliminary definitions}

Information measures are important both for the foundation of information sciences and for practical applications of information processing. They are of outmost importance in several areas of physics, beginning of course with statistical physics. Entropies and Fisher information have been identified as useful and versatile tools for characterizing complex systems, see e.g. [1, 2, 3]. Classical information theoretic inequalities, as described for instance in [4, 5], interrelate information measures. These inequalities have proved to be useful for communication theoretic problems and engineering applications. They are also connected to uncertainty relations in physics, see e.g. [6, 7, 8], and to functional inequalities in mathematics. The aim of the paper is to present and study two generalized Fisher information measures that fit well in the context of nonextensive thermostatistics, and to describe the interplay between information measures and generalized $q$-Gaussians, thus providing new characterizations of these generalized $q$-Gaussians. We first recall the context, our notations and main definitions.

\section{Generalized entropies}

The Boltzmann entropy, linked to the Shannon entropy of information theory, is at the heart of thermodynamics. For energy constraints, it is well-known that the maximum entropy distribution is the Gaussian distribution. For the analysis of complex systems, generalized entropies, which reduces to the standard one as a particular case, have been proposed. In particular, the nonextensive thermodynamics derived from Tsallis entropy, has received a high attention. There is a wide variety of applications where experiments, numerical results and analytical derivations fairly agree with the new formalisms [9]. Some physical applications of the generalized entropies, including statistics of cosmic rays, defect turbulence, optical lattices, systems with long-range interactions, superstatistical systems, etc., can be found in the recent review [10] and references therein. Let us recall here that if $x$ is a vector of $\Omega \subseteq \mathbb{R}^{n}$, and $f(x)$ a probability density defined with respect to the Lebesgue measure, then following [11], the information generating function is the quantity

$$
M_{q}[f]=\int_{\Omega} f(x)^{q} \mathrm{~d} x
$$


for $q \geq 0$. This quantity is also sometimes called "entropic moment". The Tsallis and Rényi entropies are respectively given by

$$
\begin{aligned}
& S_{q}[f]=\frac{1}{1-q}\left(M_{q}[f]-1\right), \\
& H_{q}[f]=\frac{1}{1-q} \log M_{q}[f] .
\end{aligned}
$$

For our purposes, it is not always necessary to distinguish between the two entropies that we will call collectively $q$-entropies. Finally, we will also call "entropy power" of order $q$, or $q$-entropy power the quantity

$$
N_{q}[f]=\exp \left(\frac{2}{n} H_{q}[f]\right)=M_{q}[f]^{\frac{2}{n} \frac{1}{1-q}}=\left(\int_{\Omega} f(x)^{q} \mathrm{~d} x\right)^{\frac{2}{n} \frac{1}{1-q}},
$$

for $q \neq 1$. For $q=1$, we let $N_{q}[f]=\exp \left(\frac{2}{n} H_{1}[f]\right)$, where $H_{1}[f]$ is the Boltzmann-Shannon entropy.

\section{Generalized q-Gaussian distributions}

In the context of nonextensive thermostatistics, the role devoted to the standard Gaussian distribution is extended to generalized $q$-Gaussian distributions, which include the standard Gaussian as a special case. These generalized $q$-Gaussian distributions form a versatile family that can describe problems with compact support as well as problems with heavy tailed distributions. They are also analytical solutions of actual physical problems, see e.g. [12, 13, 14], and are sometimes known as Barenblatt-Pattle functions, following their identification by [15, 16]. We shall also mention that the generalized $q$-Gaussian distributions appear in other fields, namely as the solution of non-linear diffusion equations, or as the distributions that saturate some sharp inequalities in functional analysis [17, 18, 19, 20].

Definition 1. Let $x$ be a random vector of $\mathbb{R}^{n}$, and let $|x|$ denote its Euclidean norm. For $\alpha \in(0, \infty), \gamma$ a real positive parameter and $q>(n-\alpha) / n$, the generalized Gaussian with parameter $\gamma$ has the radially symmetric probability density

$$
G_{\gamma}(x)= \begin{cases}\frac{1}{Z(\gamma)}\left(1-(q-1) \gamma|x|^{\alpha}\right)_{+}^{\frac{1}{q-1}} & \text { for } q \neq 1 \\ \frac{1}{Z(\gamma)} \exp \left(-\gamma|x|^{\alpha}\right) & \text { if } q=1\end{cases}
$$

where we use the notation $(x)_{+}=\max \{x, 0\}$, and where $Z(\gamma)$ is the partition 
function such that $G_{\gamma}(x)$ integrates to one. Its expression is given by

$$
Z(\gamma)=\frac{1}{\alpha}(\gamma)^{-\frac{n}{\alpha}} n \omega_{n} \times \begin{cases}(1-q)^{-\frac{n}{\alpha}} B\left(\frac{n}{\alpha},-\frac{1}{q-1}-\frac{n}{\alpha}\right) & \text { for } 1-\frac{\alpha}{n}<q<1 \\ (q-1)^{-\frac{n}{\alpha}} B\left(\frac{n}{\alpha}, \frac{1}{q-1}+1\right) & \text { for } q>1 \\ \Gamma\left(\frac{n}{\alpha}\right) & \text { if } q=1\end{cases}
$$

where $B(x, y)$ denotes the beta function and $\omega_{n}=\pi^{\frac{n}{2}} / \Gamma\left(\frac{n}{2}+1\right)$ is the volume of the $n$-dimensional unit ball. The parameter $\gamma$ is linked to the moments of the density. For instance, if $m_{\alpha}\left[G_{\gamma}\right]$ denotes the moment of order $\alpha$, we have $\gamma^{-1}=\left(\left(1+\frac{\alpha}{n}\right) q-1\right) m_{\alpha}\left[G_{\gamma}\right]$, for $q>n /(n+\alpha)$. We may also note that $\gamma^{-\frac{1}{\alpha}}$ is a scale parameter, e.g. we have $Z(\gamma)=\gamma^{-\frac{1}{\alpha}} Z(1)$. Usually, the term $q$-Gaussian corresponds to the case $\alpha=2$ above. The generalized $q$ Gaussians are sometimes called stretched $q$-Gaussians, where $\alpha$ is a stretch parameter.

In the sequel, we will denote $G$ the generalized Gaussian obtained with $\gamma=1$. For $q>1$, the density has a compact support, while for $q \leq 1$ it is defined on the whole $\mathbb{R}^{n}$ and behaves as a power distribution for $|x| \rightarrow \infty$. A shorthand notation for the expression of the generalized $q$-Gaussian density is

$$
G_{\gamma}(x)=\frac{1}{Z(\gamma)} \exp _{q^{*}}\left(-\gamma|x|^{\alpha}\right)
$$

with $q^{*}=2-q$, and where the so-called $q$-exponential function, and its inverse the $q$-logarithm, are given by

$$
\begin{gathered}
\exp _{q}(x):=(1+(1-q) x)_{+}^{\frac{1}{1-q}} \text { for } q \neq 1 \text { and } \exp _{q=1}(x):=\exp (x), \\
\ln _{q}(x):=\frac{x^{1-q}-1}{1-q} \text { for } q \neq 1 \text { and } \ln _{q=1}(x):=\ln (x) .
\end{gathered}
$$

It is well known that the Gaussian distribution is a central distribution with respect to classical information measures and inequalities. In particular, the Gaussian distribution is both a maximum entropy and a minimum Fisher information distribution over all distributions with the same variance. We will see that the same kind of result holds for the family of generalized $q$ Gaussians, for Rényi or Tsallis entropy and suitable extensions of the Fisher information.

\section{Generalized $(\beta, q)$-Fisher information}

In the context of nonextensive thermostatistics, several authors have introduced and studied generalized versions of the Fisher information. Among 
these contributions we note the series of papers [21, 22, 23, 24], and the proposals of Naudts [25, 26] and Furuichi [27, 28], which are related to the generalized Fisher information that are used here - see [29] for a discussion of these links.

In our recent work [29, 30], we have thrown a bridge between concepts in estimation theory and tools of nonextensive thermostatistics. Using the notion of escort distribution, we have introduced a generalized Fisher information and established an extended version of the Cramér-Rao inequality for parameter estimation. In the case of a location parameter, it reduces to an extended version of the standard Cramér-Rao inequality, which is saturated by the generalized $q$-Gaussians. A closely related generalized Fisher information has been originally introduced by Lutwak et al. [31] in information theory and extended to the multidimensional in [32] and independently in [33]. These two extensions of the Fisher information measure are defined as follows.

Definition 2. Let $f(x)$ be a probability density function defined over a subset $\Omega$ of $\mathbb{R}^{n}$. Let $|x|$ denote the Euclidean norm of $x$ and $\nabla f$ the gradient operator. If $f(x)$ is continuously differentiable over $\Omega$, then for $q \geq 0, \beta>1$, the generalized $(\beta, q)$-Fisher information is defined by

$$
\begin{aligned}
\phi_{\beta, q}[f] & =\int_{\Omega} f(x)^{\beta(q-1)+1}\left(\frac{|\nabla f(x)|}{f(x)}\right)^{\beta} \mathrm{d} x \\
& =E\left[f(x)^{\beta(q-1)}|\nabla \ln f(x)|^{\beta}\right]=E\left[\left|\nabla \ln _{q *} f(x)\right|^{\beta}\right]
\end{aligned}
$$

with $q_{*}=2-q$.

With the same notations and assumptions as above, the second generalized $(\beta, q)$-Fisher information is

$$
I_{\beta, q}[f]=\left(\frac{q}{M_{q}[f]}\right)^{\beta} \phi_{\beta, q}[f]=\left(\frac{q}{M_{q}[f]}\right)^{\beta} E\left[\left|\nabla \ln _{q *} f(x)\right|^{\beta}\right] .
$$

These two extensions of the Fisher information measure, the $(\beta, q)$-Fisher information $\phi_{\beta, q}[f]$ and $I_{\beta, q}[f]$, depend on an entropic index $q$ and on a parameter $\beta$. As mentioned above, the $\phi_{\beta, q}[f]$ form has been introduced by Lutwak et al. [31] in information theory and is related to general results in functional analysis. The second form of generalized Fisher information has been introduced in the derivation of a general Cramér-Rao inequality for parameter estimation [29, 30]. These two information only differ by a prefactor, but actually originates from two different settings and lead to different, 
though similar, inequalities and characterizations. Of course, both information reduce to the standard Fisher information in the case $q=1, \beta=2$. We will see that these two generalized $(\beta, q)$-Fisher information interplay nicely with $q$-entropies and generalized $q$-Gaussians, generalizing classical information relations, and thus allow a natural extension of the usual ShannonFisher-Gaussian setting.

\section{Structure and contributions of the paper}

As already indicated, the goal of the paper is to introduce two generalized Fisher information in the context of nonextensive thermostatistics, to document their main properties and study the interplay between generalized information measures and generalized $q$-Gaussians. Though the paper reviews some of our previous results, most of the results presented here are new. The paper is organized into two main parts. In the first part, we present several variational characterizations of the generalized $q$-Gaussian distributions, where the generalized Fisher information play a fundamental role. The new findings include (i) the second generalized Stam inequality (14) which lower bounds the product of the entropy power and of the extended Fisher information, (ii) the definition of several information functionals minimized by the generalized $q$-Gaussians, (iii) the derivation of the extended de Bruijn inequality (40, 41) and of the de Bruijn entropy power inequality (42) which intimately links the $q$-entropies to the generalized Fisher information through a doubly nonlinear diffusion equation. In the second section, we establish several properties of the generalized Fisher information which might be useful for thermodynamics considerations. The discussion includes the additivity and mixing properties of the generalized Fisher information, as well as an original derivation of the preservation of the Legendre structure.

\section{Variational characterizations of generalized $q$-Gaussians and gen- eralized Fisher information}

It is known that the $q$-Gaussians maximize the Tsallis or Renyi entropy among all probability distributions with a given variance or covariance [34]. This means that the $q$-Gaussians are the canonical distributions of the generalized thermostatistics based on Tsallis or Rényi entropy. The maximum

entropy formalism directly induces a variational characterization of generalized $q$-Gaussians as the extremal functions of the Lagrangian

$$
L[f]=H_{q}[f]+\mu m_{\alpha}[f],
$$


where $\mu$ is a Lagrange multiplier and $m_{\alpha}[f]$ denotes the moment of order $\alpha$ of the probability distribution $f$. A remarkable point is that the generalized $q$-Gaussians are also solutions of other variational problems as well, and that these problems involve the generalized $(\beta, q)$-Fisher information we defined above. Such characterizations are described now.

\subsection{The minimum of generalized Fisher information among distributions with a given q-entropy is attained for generalized q-Gaussians}

Another characterization of the generalized $q$-Gaussian is indeed the fact that they minimize the extended Fisher information among all distributions with a given $q$-entropy. This result is a consequence of a generalized Stam inequality which lower bounds a product of the entropy power and of the generalized Fisher information. This inequality is stated in the Proposition below and has been established in [31] in the monodimensional case, in [32] and [33] in the multidimensional case, and in [30] for arbitrary norms. The statement of case $(b)$ is new.

Proposition 1. [Generalized Stam inequalities] For $n \geq 1, \beta$ and $\alpha$ Hölder conjugates of each other, $\alpha>1$, and $q>\max \{(n-1) / n, n /(n+\alpha)\}$, then for any probability density on $\mathbb{R}^{n}$, supposed continuously differentiable, the following generalized Stam inequalities hold

$$
\begin{aligned}
& \text { (a) } \phi_{\beta, q}[f]^{\frac{1}{\beta}} N_{q}[f]^{\frac{\lambda}{2}} \geq \phi_{\beta, q}[G]^{\frac{1}{\beta}} N_{q}[G]^{\frac{\lambda}{2}}, \\
& \text { (b) } I_{\beta, q}[f]^{\frac{1}{\beta}} N_{q}[f]^{\frac{1}{2}} \geq I_{\beta, q}[G]^{\frac{1}{\beta}} N_{q}[G]^{\frac{1}{2}} .
\end{aligned}
$$

with

$$
\lambda=n(q-1)+1>0
$$

and with equality if and only if $f$ is any generalized q-Gaussian (7).

In the previous expressions, $\phi_{\beta, q}[G], I_{\beta, q}[G]$ and $N_{q}[G]$ are the values taken by the $(\beta, q)$-Fisher information and the entropy power when the probability density $f$ is the generalized $q$-Gaussian $G$. The exact expressions of these quantities are given in the Appendix, section 5 .

Proof. The inequality (a) is proved in [32] and [33] using a general sharp Gagliardo-Nirenberg inequality due to Cordero et al. [19]. It is quite easy to get the same kind of generalized Stam inequality for the second generalized Fisher information. Indeed, for the inequality (b), it suffices to replace $\phi_{\beta, q}[f]^{\frac{1}{\beta}}$ in $(13)$ by $I_{\beta, q}[f]^{\frac{1}{\beta}} M_{q}[f] / q$, to use the fact that $M_{q}[f]=$ $N_{q}[f]^{\frac{n}{2}(1-q)}$ and to simplify the exponent to obtain (14). 
The generalized Stam inequalities imply that the generalized $q$-Gaussian minimize the extended Fisher information within the set of probability distributions with a fixed $q$-entropy power: $G_{\gamma}$ is the solution of

$$
\inf _{f}\left\{\phi_{\beta, q}[f] \text { or } I_{\beta, q}[f]: f \in \mathcal{P}, N_{q}[f]=N_{q}\left[G_{\gamma}\right]\right\},
$$

where $\mathcal{P}$ is the probability simplex. In turn, this leads to the following variational description.

Proposition 2. The generalized q-Gaussian $G$ minimize the following two information functionals

$$
\begin{aligned}
& J_{1}[f]=\frac{1}{\beta \lambda} \phi_{\beta, q}[f] N_{q}[G]+\frac{1}{2} \phi_{\beta, q}[G] N_{q}[f] \\
& J_{2}[f]=\frac{1}{\beta} I_{\beta, q}[f] N_{q}[G]+\frac{1}{2} I_{\beta, q}[G] N_{q}[f]
\end{aligned}
$$

with $\lambda$ given by (15).

Another statement of this result could be in terms of an energy functional. Let $f=u^{k}$, with $k=\beta /(\beta(q-1)+1)$. With this notation, the generalized Fisher information reduces to

$$
\phi_{\beta, q}[f]=|k|^{\beta} \int_{\Omega}|\nabla u(x)|^{\beta} \mathrm{d} x,
$$

which is the $\beta$-Dirichlet energy of function $k u(x)$. In addition, $M_{q}[f]=$ $\int_{0} u(x)^{k q} \mathrm{~d} x$ is a measure of informational energy of order $k q$ in the sense of [35, 36]. Then, (17) becomes

$$
J_{1}[f]=\frac{1}{\beta \lambda} N_{q}[G] \int_{\Omega}|\nabla u(x)|^{\beta} \mathrm{d} x+\frac{1}{2} \phi_{\beta, q}[G]\left(\int_{\Omega} u(x)^{k q} \mathrm{~d} x\right)^{\frac{1}{1-q}}
$$

a weighted sum of these two energies.

Proof. Consider the first generalized Stam inequality. As already indicated, a direct consequence of this inequality is that the generalized $q$-Gaussian $G_{\gamma}$ solves problem (16). The Lagrangian corresponding to this problem is

$$
J(f)=\phi_{\beta, q}[f]+\mu N_{q}[f] .
$$

This Lagrangian is minimum for a $q$-Gaussian $G_{\gamma}$ with parameter $\gamma$. In the Lagrangian, for a fixed parameter $\gamma$, the Lagrange parameter $\mu$ is a function of $\gamma$. Suppose that $\mu$ is chosen such that the optimum distribution is the 
generalized $q$-Gaussian with $\gamma=1$. In such conditions, we have $J\left(G_{\gamma}\right) \geq$ $J(G), \forall \gamma$. On the other hand, we have the following scaling identities:

$$
\left\{\begin{array}{l}
M_{q}\left[G_{\gamma}\right]=\gamma^{\frac{n}{\alpha}(q-1)} M_{q}[G], \\
\phi_{\beta, q}\left[G_{\gamma}\right]=\gamma^{\frac{\beta \lambda}{\alpha}} \phi_{\beta, q}[G], \\
I_{\beta, q}\left[G_{\gamma}\right]=\gamma^{\frac{\beta}{\alpha}} I_{\beta, q}[G], \\
Z(\gamma)=\gamma^{-\frac{n}{\alpha}} Z(1)
\end{array}\right.
$$

with $\lambda$ given by (15). Thus, the inequality $J\left(G_{\gamma}\right) \geq J(G)$ means that

$$
J\left(G_{\gamma}\right)=\gamma^{\frac{\beta \lambda}{\alpha}} \phi_{\beta, q}[G]+\mu \gamma^{-\frac{2}{\alpha}} N_{q}[G]
$$

is minimum in $\gamma$ for $\gamma=1$. The derivative with respect to $\gamma$ then vanishes at $\gamma=1$, and this yields the value

$$
\mu=\frac{\beta \lambda}{2} \frac{\phi_{\beta, q}[G]}{N_{q}[G]} .
$$

Then, it only remains to set $J_{1}[f]=\frac{N_{q}[G]}{\beta \lambda} J[f]$ to obtain (17). The second information functional (18) is established in the same way.

\subsection{The minimum of generalized Fisher information among distributions with a given moment is attained for generalized q-Gaussians}

In addition to be the maximum entropy distribution in the set of all distributions with a given variance, it is well-known that the Gaussian distribution is also a minimum Fisher information distribution over all distributions with the same variance. This can be seen as a consequence of the CramérRao inequality for a location parameter. The same kind of characterization holds in the multidimensional case for the generalized $q$-Gaussian and the generalized Fisher information.

In [31, 32, 33], a Cramér-Rao inequality for generalized $q$-Gaussian involving the $(\beta, q)$-Fisher information $\phi_{\beta, q}[f]$ has been established. The $(\beta, q)$ Fisher information $I_{\beta, q}[f]$ has been introduced in the context of parameter estimation in [29, 30] and a related Cramér-Rao inequality proved. These inequalities mean that among all distributions with a given moment, the generalized $q$-Gaussians are the minimizers of extended versions of the Fisher information, just as the standard Gaussian minimizes Fisher information over all distributions with a given variance. We begin by recalling the generalized Cramér-Rao inequalities involving the generalized Fisher information measures and saturated by the generalized $q$-Gaussians. Then, we show that the generalized $q$-Gaussians can also be characterized as the minimizers of two new variational problems. 
Proposition 3. [Generalized Cramér-Rao inequalities] For $n \geq 1, \beta$ and $\alpha$ Hölder conjugates of each other, $\alpha>1, q>\max \{(n-1) / n, n /(n+\alpha)\}$ then for any probability density $f$ on $\Omega \subseteq \mathbb{R}^{n}$, supposed continuously differentiable and such that the involved information measures are finite,

$$
\begin{aligned}
m_{\alpha}[f]^{\frac{1}{\alpha}} \phi_{\beta, q}[f]^{\frac{1}{\beta \lambda}} \geq m_{\alpha}[G]^{\frac{1}{\alpha}} \phi_{\beta, q}[G]^{\frac{1}{\beta \lambda}} \\
m_{\alpha}[f]^{\frac{1}{\alpha}} I_{\beta, q}[f]^{\frac{1}{\beta}} \geq m_{\alpha}[G]^{\frac{1}{\alpha}} I_{\beta, q}[G]^{\frac{1}{\beta}}=n
\end{aligned}
$$

with $\lambda=n(q-1)+1$, where the general $(\beta, q)$-Fisher information are defined in (10) and (12) and where the equality holds iff $f$ is a generalized Gaussian $g=G_{\gamma}$.

Proof. The proof of (25) can be found in [32] and [33] in the multidimensional case. As far as (26) is concerned, the proof is given in [29, 30] as a particular case of the more general Cramér-Rao inequality for parameter estimation. The fact that the lower bound $m_{\alpha}[G]^{\frac{1}{\alpha}} I_{\beta, q}[G]^{\frac{1}{\beta}}$ is exactly equal to $n$ is a direct consequence of the proof of (26).

The Cramér-Rao inequalities (25)-(26) imply that $G_{\gamma}$ realizes the minimum of the generalized Fisher information in the set of all probability densities with a given moment of order $\alpha$ :

$$
\inf _{f}\left\{\phi_{\beta, q}[f] \text { or } I_{\beta, q}[f]: f \in \mathcal{P}, m_{\alpha}[f]=m_{\alpha}\left[G_{\gamma}\right]\right\},
$$

where $\mathcal{P}$ is the probability simplex. As above and as a direct consequence, we obtain a variational characterization of the generalized $q$-Gaussians:

Proposition 4. The generalized q-Gaussian $G$ minimize the following two information functionals

$$
\begin{aligned}
& J_{3}[f]=\frac{1}{\beta \lambda} \phi_{\beta, q}[f] m_{\alpha}[G]+\frac{1}{\alpha} \phi_{\beta, q}[G] m_{\alpha}[f] \\
& J_{4}[f]=\frac{1}{\beta} I_{\beta, q}[f] m_{\alpha}[G]+\frac{1}{\alpha} I_{\beta, q}[G] m_{\alpha}[f]
\end{aligned}
$$

Proof. The proof proceeds just as the proof of Proposition 2, Let us consider the first Cramér-Rao inequality (25) and the problem (27). The corresponding Lagrangian is

$$
J(f)=\phi_{\beta, q}[f]+\mu m_{\alpha}[f] .
$$

Assume that $\mu$ is chosen such that the optimum distribution is the generalized $q$-Gaussian with $\gamma=1$. In such conditions, we have $J\left(G_{\gamma}\right) \geq J(G)$, 
$\forall \gamma$. By the scaling identities (22) and the additional fact that $m_{\alpha}\left[G_{\gamma}\right]=$ $\gamma^{-1} m_{\alpha}[G]$, we get that

$$
J\left(G_{\gamma}\right)=\gamma^{\frac{\beta \lambda}{\alpha}} \phi_{\beta, q}[G]+\mu \gamma^{-1} m_{\alpha}[G] .
$$

Since this functional is minimum for $\gamma=1$, its derivative vanishes at $\gamma=1$, which gives

$$
\mu=\frac{\beta \lambda}{\alpha} \frac{\phi_{\beta, q}[G]}{m_{\alpha}[G]}
$$

and in turn the inequality (28). The second information functional (29) is established in the same way.

We shall note that up to the change of function $u(x)^{k}=f(x)$, the EulerLagrange equation associated with the two functionals (28) and (29) is a $\beta$-Laplace equation of the form

$$
\triangle_{\beta} u(x)-\frac{1}{\alpha k^{\beta-1}} \frac{\phi_{\beta, q}[G]}{m_{\alpha}[G]}|x|^{\alpha} u(x)^{k-1}=0,
$$

where we used the $\beta$-Laplacian operator $\Delta_{\beta} u:=\operatorname{div}\left(|\nabla u|^{\beta-2} \nabla u\right)$. In the monodimensional case and if $\beta=2$, we obtain an instance of the generalized Emden-Fowler equation $u^{\prime \prime}(x)+h(x) u(x)^{\gamma}=0$, where $h(x)$ is a given function. This kind of equations arises in studies of gaseous dynamics in astrophysics, in certain problems in fluid mechanics and pseudoplastic flow, see [37], as well as in some reaction-diffusion processes.

\subsection{The derivative of q-entropies give the generalized Fisher information -} An extended de Bruijn identity

A fundamental connection between the Boltzmann-Shannon entropy, Fisher information, and the Gaussian distribution is given by the de Bruijn identity [6]. We show here that this important connection can be extended to the $q$-entropies, the generalized Fisher information and the generalized $q$ Gaussians.

The de Bruijn identity states that if $Y_{t}=X+\sqrt{2 t} Z$ where $Z$ is a standard Gaussian vector and $X$ a random vector of $\mathbb{R}^{n}$, independent of $Z$, then

$$
\frac{\mathrm{d}}{\mathrm{d} t} H\left[f_{Y_{t}}\right]=I_{2,1}\left[f_{Y_{t}}\right]=\phi_{2,1}\left[f_{Y_{t}}\right]
$$

where $f_{Y_{t}}$ denotes the density of $Y_{t}=X+\sqrt{2 t} Z$. In thermodynamics, this can be seen as a consequence of the second law for an isolated system out of 
equilibrium. The standard proof of the de Bruijn identity uses the fact that $Y_{t}$ satisfies the well-known heat equation [38]

$$
\frac{\partial f}{\partial t}=\Delta f
$$

where $\Delta$ denotes the Laplace operator.

It is possible to consider more general versions of the heat equation. In particular, it is known that the porous medium equation, or the fast diffusion equation, admit $q$-Gaussians as solutions. This connects the $q$-Gaussian distributions to differential equations of physics, and has been noticed in the nonextensive context, see e.g. [13, 39]. The porous medium equation and fast diffusion equation correspond to the differential equation

$$
\frac{\partial f}{\partial t}=\Delta f^{m}
$$

with $m>1$ for the porous medium equation and $<1$ for the fast diffusion. These two equations have been exhaustively studied and characterized by J. L. Vazquez, e.g. in the two books [40, 41]. These equations appear in a large number of physical situations, including fluid mechanics, nonlinear heat transfer or diffusion. Other applications have been reported in mathematical biology, lubrification, boundary layer theory, etc, see the series of applications presented in [40, chapters 2 and 21] and references therein.

The porous medium equation (36) can be generalized to a wider form, the doubly nonlinear equation, which involves a $p$-Laplacian operator $\Delta_{p} f:=$ $\operatorname{div}\left(|\nabla f|^{p-2} \nabla f\right)$, and the power $m$ of the porous medium or fast diffusion equation. This doubly nonlinear equation takes the form

$$
\frac{\partial}{\partial t} f=\Delta_{\beta} f^{m}=\operatorname{div}\left(\left|\nabla f^{m}\right|^{\beta-2} \nabla f^{m}\right)
$$

where we use $p=\beta$ for convenience and coherence with the other notations in the paper. The $\beta$-Laplacian operator typically in the minimization of a Dirichlet energy like $\int|\nabla f|^{\beta} \mathrm{d} x$ which leads to the Euler-Lagrange equation $\Delta_{\beta} f=0$. A discussion on the $p$-Laplace equation can be found in [42].

As we can see, the doubly nonlinear equation includes the standard heat equation $(\beta=2, m=1)$, the $\beta$-Laplace equation $(\beta \neq 2, m=1)$, the porous medium equation $(\beta=2, m>1)$ and the fast diffusion equation $(\beta=2$, $m<1)$. It can be shown, see [41, page 192], that for $m(\beta-1)+(\beta / n)-1>0$, (37) has a unique self-similar solution, whose initial value is the Dirac mass 
at the origin. This fundamental solution is given by

$$
f(x, t)=\frac{1}{t^{\frac{n}{\delta}}} B\left(\frac{x}{t^{\frac{1}{\delta}}}\right), \text { with } B(x)= \begin{cases}\left(C-k|x|^{\alpha}\right)_{+}^{\frac{\beta-1}{m(\beta-1)-1}} & \text { for } m \neq \frac{1}{\beta-1} \\ \frac{1}{\sigma} \exp \left(-\frac{|\beta-1|}{\beta^{\alpha}}|x|^{\alpha}\right) & \text { for } m=\frac{1}{\beta-1}\end{cases}
$$

with

$$
\delta=n(\beta-1) m+\beta-n>0, \quad k=\frac{m(\beta-1)-1}{\beta}\left(\frac{1}{\delta}\right)^{\frac{1}{\beta-1}} \text { and } \alpha=\frac{\beta}{\beta-1} .
$$

The constants $C$ and $\sigma$ are uniquely determined by mass conservation, e.g. $\int f(x, t) \mathrm{d} x=1$. Of course, we observe that the function $B(x)$ above is analog to the generalized $q$-Gaussian (7). In the literature, $B(x)$ is called the Barenblatt profile following its identification as a solution for heat release from a point source [15, 16].

The doubly nonlinear diffusion equation also enables to derive a nice extension of the de Bruijn identity (34). A remarkable point is that the extended $(\beta, q)$-Fisher information naturally pops up in this identity, generalizing the role of the standard Fisher information in the classical de Bruijn identity. This is stated in the next Proposition. It shall be mentioned that the case $\beta=2$ of this result has been given in a nice paper by Johnson and Vignat [34].

Proposition 5. [Extended de Bruijn identity] Let $f(x, t)$ a probability distributions satisfying the doubly nonlinear equation (37). Assume that the domain $\Omega$ is independent of $t$, that $f(x, t)$ is differentiable with respect to $t$, and that $\frac{\partial}{\partial t} f(x, t)^{q}$ is absolutely integrable and locally integrable with respect to $t$. Then, for $q=m+1-\frac{\alpha}{\beta}, \lambda=n(q-1)+1$, and $\delta=\beta \lambda-n(q-1)$, we have

$$
\begin{aligned}
\frac{d}{d t} H_{q}[f] & =\frac{q m^{\beta-1}}{M_{q}[f]} \phi_{\beta, q}[f]=\left(\frac{m}{q}\right)^{\beta-1} M_{q}[f]^{\beta-1} I_{\beta, q}[f] . \\
\frac{d}{d t} S_{q}[f] & =q m^{\beta-1} \phi_{\beta, q}[f]=\left(\frac{m}{q}\right)^{\beta-1} M_{q}[f]^{\beta} I_{\beta, q}[f] .
\end{aligned}
$$

where $M_{q}[f]=\int f^{q}$ and where $H_{q}[f]=\frac{1}{1-q} \log M_{q}[f]$ is the Rényi entropy and $S_{q}[f]=\frac{1}{1-q}\left(M_{q}[f]-1\right)$ the Tsallis entropy. In terms of the entropy power $N_{q}[f]=M_{q}[f]^{\frac{2}{n} \frac{1}{1-q}}$ we also have

$$
\frac{d}{d t} N_{q}[f]^{\frac{\delta}{2}}=q \frac{\delta}{n} m^{\beta-1} N_{q}[f]^{\frac{\beta \lambda}{2}} \phi_{\beta, q}[f]=\frac{\delta}{n}\left(\frac{m}{q}\right)^{\beta-1} N_{q}[f]^{\frac{\beta}{2}} I_{\beta, q}[f],
$$


and

$$
\frac{d}{d t} N_{q}[f]^{\frac{\delta}{2}} \geq \frac{d}{d t} N_{q}[G]^{\frac{\delta}{2}}
$$

Of course, the classical de Bruijn identity

$$
\frac{\mathrm{d}}{\mathrm{d} t} H[f]=\phi_{2,1}[f]=I_{2,1}[f] .
$$

is recovered from the extended de Bruijn identity (40), for $\alpha=\beta=2$, and $q=m=1$. We recognize in the right side of (42) the very same products of entropy power and $(\beta, q)$-Fisher information that appear in the the generalized Stam inequalities (13) and (14). By these inequalities, we obtain (43) which indicates that the derivative of the entropy power in minimum for the generalized $q$-Gaussian, with equality if and only if $f=G_{\gamma}, \forall \gamma$. Furthermore, it is known that the solutions of the doubly nonlinear equation converge to the Barenblatt profile [41]. Thus, we see that the minimum of the derivative of the entropy power is always attained asymptotically. In a very recent and nice paper [43], Savaré and Toscani have shown that in the case $\beta=2$, $m=q$, the entropy power $N_{q}[f]^{\frac{\delta}{2}}$ is a concave function of $t$. A consequence of this result is that the derivative (42) of entropy power is non increasing in time, with a minimum for $f=G$, reached at least asymptotically. It would be very interesting to get the same kind of result in the present setting. For now, let us proceed with the proof of extended de Bruijn identity.

Proof. Let us consider the Rényi entropy

$$
H_{q}[f]=\frac{1}{1-q} \log \int_{\Omega} f(x, t)^{q} \mathrm{~d} x .
$$

The regularity assumptions in the statement of the proposition enable to use Leibnitz' rule and differentiate under the integral sign:

$$
\begin{aligned}
(1-q) \frac{\partial}{\partial t} H_{q}[f] & =\frac{1}{\int_{\Omega} f(x, t)^{q} \mathrm{~d} x} \int_{\Omega} \frac{\partial}{\partial t} f(x, t)^{q} \mathrm{~d} x=\frac{q}{\int f(x, t)^{q} \mathrm{~d} x} \int_{\Omega} f(x, t)^{q-1} \frac{\partial}{\partial t} f(x, t) \mathrm{d} x \\
= & \frac{q}{\int_{\Omega} f(x, t)^{q} \mathrm{~d} x} \int_{\Omega} f(x, t)^{q-1} \Delta_{\beta} f(x, t)^{m} \mathrm{~d} x \\
= & \frac{q}{\int_{\Omega} f(x, t)^{q} \mathrm{~d} x} \int_{\Omega} f(x, t)^{q-1} \nabla \cdot\left[\left|\nabla f^{m}\right|^{\beta-2} \nabla f^{m}\right] \mathrm{d} x
\end{aligned}
$$

where we used the fact that $f(x, t)$ satisfies the doubly nonlinear heat equation (37). By the divergence theorem and the product rule, we have

$$
\int_{U} \nabla \cdot(\psi A) d V=\int_{\partial U} \psi A . \eta d S=\int_{U} \psi \nabla \cdot A d V+\int_{U} A . \nabla \psi d V
$$


where $\eta$ is a unit vector orthogonal to the hypersurface $\partial U$. Thus, considering

$$
\int_{\Omega} f(x, t)^{q-1} \Delta_{\beta} f(x, t)^{m} \mathrm{~d} x=\int_{\Omega} f(x, t)^{q-1} \nabla \cdot\left[\left|\nabla f^{m}\right|^{\beta-2} \nabla f^{m}\right] \mathrm{d} x,
$$

we get, with $\psi=f(x, t)^{q-1}$ and $A=\left|\nabla f^{m}\right|^{\beta-2} \nabla f^{m}$,

$$
\begin{array}{r}
\int_{\Omega} f(x, t)^{q-1} \nabla \cdot\left[\left|\nabla f^{m}\right|^{\beta-2} \nabla f^{m}\right] \mathrm{d} x=\int_{\partial \Omega} f(x, t)^{q-1}\left|\nabla f^{m}\right|^{\beta-2} \nabla f^{m} \cdot \eta \mathrm{d} x \\
-\int_{\Omega}(q-1) f(x, t)^{q-2} \nabla f \cdot\left[\left|\nabla f^{m}\right|^{\beta-2} \nabla f^{m}\right] \mathrm{d} x . \quad(51)
\end{array}
$$

Assuming that both $f(x, t)$ and $|\nabla f|$ tend to zero on the boundary $\partial \Omega$ of the domain, it remains

$$
\begin{aligned}
\int_{\Omega} f(x, t)^{q-1} \nabla \cdot\left[\left|\nabla f^{m}\right|^{\beta-2} \nabla f^{m}\right] \mathrm{d} x & =-(q-1) \int_{\Omega} f(x, t)^{q-2} \nabla f \cdot\left[\left|\nabla f^{m}\right|^{\beta-2} \nabla f^{m}\right] \mathrm{d} x, \\
& =-(q-1) m^{\beta-1} \int_{\Omega} f(x, t)^{\beta m+q-m-1}\left(\frac{|\nabla f|}{f}\right)^{\beta} \mathrm{d} x,
\end{aligned}
$$

where we used twice the fact that $\nabla f^{m}=m f^{m-1} \nabla f$. Finally, if we choose $q=m+1-\frac{\alpha}{\beta}$, the exponent in the last equation reduces to $\beta(q-1)+1$, and

$$
\begin{aligned}
\frac{\partial}{\partial t} H_{q}[f] & =\frac{q m^{\beta-1}}{\int f(x)^{q} \mathrm{~d} x} \int_{\Omega} f(x, t)^{\beta(q-1)+1}\left(\frac{|\nabla f|}{f}\right)^{\beta} \mathrm{d} x \\
& =\frac{q m^{\beta-1}}{M_{q}[f]} \phi_{\beta, q}[f] .
\end{aligned}
$$

Using the relationship (12) between $I_{\beta, q}[f]$ and $\phi_{\beta, q}[f]$, this identity becomes

$$
\frac{\partial}{\partial t} H_{q}[f]=\left(\frac{m}{q}\right)^{\beta-1} M_{q}[f]^{\beta-1} I_{\beta, q}[f] .
$$

Of course, the second inequality (41) follows immediately. If we consider the entropy power $N_{q}=M_{q}^{\frac{2}{n} \frac{1}{1-q}}$ instead of the entropy, we obtain along similar steps the following relation:

$$
\frac{\partial}{\partial t} N_{q}[f]^{\frac{\delta}{2}}=q \frac{\delta}{n} m^{\beta-1} N_{q}[f]^{\frac{\beta \lambda}{2}} \phi_{\beta, q}[f]=\frac{\delta}{n}\left(\frac{m}{q}\right)^{\beta-1} N_{q}[f]^{\frac{\beta}{2}} I_{\beta, q}[f] .
$$

with $\lambda=n(q-1)+1$ and $\delta=n(\beta-1)(q-1)+\beta=\beta \lambda-n(q-1)$. 


\section{Thermodynamics considerations}

Given an information measure or a generalized entropy, it is possible to investigate whether it is possible to construct a thermodynamics based on this measure. Several of such constructions have been proposed, namely the nonextensive thermostatistics based on the Tsallis entropy, see the books [44, 9], the construction of thermodynamics based on the Rényi entropy, with connections with the multifractals [45, 46, 47, 48], or based on the SharmaMittal entropy [49]. Generalized entropies obtained through Beck and Cohen's superstatistics have also been considered [50, 10, 51]. Finally, following the pioneering work of Frieden, thermodynamics based on the Fisher information have been proposed [52, 53, 54, 55, 56].

Though it is not the objective of the present paper to discuss and compare the pros and cons of these different thermodynamics, these prior works naturally lead us to examine the status of some basic properties of conventional thermodynamics in the case of the generalized Fisher information. In this spirit, we consider the additivity property of the information, the mixing property, and finally the Legendre structure of thermodynamics.

\subsection{Additivity}

In classical thermodynamics, additivity is the principle that if a global system is constituted of two independent components, then the total entropy is the sum of entropies of each subsystem. However, the use of a different form of entropy or complexity measure can lead to reconsider this property. It is well known, for instance, that the Tsallis entropy is non additive. Indeed, if we use a measure of information, it is understandable that the information given by a whole system can be different of the sum of the information given by its components ; the difference representing an information gain attached to the combining of the elementary systems. In the case of Fisher information, the information is additive for independent systems. However, this property is not preserved for the generalized Fisher information as soon as $q \neq 1$ or $\beta \neq 2$. Nevertheless, we still have an interesting inequality which bounds the $(\beta, q)$-Fisher information of a combined system. Indeed, if $X$ and $Y$ are two independent random vectors with densities $f_{X}(x)$ and $f_{Y}(y)$, then the generalized Fisher information $\phi_{\beta, q}$ attached to the joint distribution $f_{X, Y}(x, y)=f_{X}(x) f_{Y}(y)$ is

$\phi_{\beta, q}\left[f_{X} f_{Y}\right]=\int\left(f_{X}(x) f_{Y}(y)\right)^{\beta(q-2)+1}\left|\nabla f_{X}(x) f_{Y}(y)+f_{X}(x) \nabla f_{Y}(y)\right|^{\beta} \mathrm{d} x \mathrm{~d} y$. 
The Minkovski inequality indicates that the triangle inequality holds in $L_{p}$ spaces, e.g. for a weighted $L_{p}$ norm

$$
\left(\int w(x)|f(x)+g(x)|^{p} \mathrm{~d} x\right)^{\frac{1}{p}} \leq\left(\int w(x)|f(x)|^{p} \mathrm{~d} x\right)^{\frac{1}{p}}+\left(\int w(x)|g(x)|^{p} \mathrm{~d} x\right)^{\frac{1}{p}}
$$

with $w(x)>0$ and $p>1$. Applying this to the generalized Fisher information (58), we immediately obtain

$$
\phi_{\beta, q}\left[f_{X} f_{Y}\right]^{\frac{1}{\beta}} \leq M_{\beta(q-1)+1}\left[f_{Y}\right]^{\frac{1}{\beta}} \phi_{\beta, q}\left[f_{X}\right]^{\frac{1}{\beta}}+M_{\beta(q-1)+1}\left[f_{X}\right]^{\frac{1}{\beta}} \phi_{\beta, q}\left[f_{Y}\right]^{\frac{1}{\beta}},
$$

where $M_{q}[f]$ is the information generating function. By the general power mean inequality, we know that $M_{a}[f]^{\frac{1}{a}} \leq M_{b}[f]^{\frac{1}{b}}$ for $a<b$. Consequently, when $q<1, M_{\beta(q-1)+1}<M_{1}=1$, and the inequality (60) reduces to

$$
\phi_{\beta, q}\left[f_{X} f_{Y}\right]^{\frac{1}{\beta}} \leq \phi_{\beta, q}\left[f_{X}\right]^{\frac{1}{\beta}}+\phi_{\beta, q}\left[f_{Y}\right]^{\frac{1}{\beta}} .
$$

\subsection{Mixing property}

It is well known that the Boltzmann entropy increases when two or more different substances are mixed. A simple interpretation is that the uncertainty on (or the complexity of) the mixed system increases and consequently its the entropy. This mixing property directly implies that the entropy is a concave function. If we substitute to the classical entropy a different form which measures differently the amount of information in a system, then the mixing property, though thermodynamically appealing, is not necessarily preserved. This is what happens in the case of our generalized Fisher information. It is known that the standard Fisher information is a convex function [57, 52]. Similarly, it has be shown that this is also the case for $q=1$ and any exponent $\beta>1$ [58]. We show here that this is in fact true for any $q \geq 1$. Consider the integrand in the definition of the $(\beta, q)$-Fisher information, and denote $x=|\nabla f|$ and $y=f$. Then, let us define

$$
h(x, y)=x^{\beta} y^{(b-\beta)},
$$

with $b=\beta(q-1)+1$. This function will be jointly convex, with respect to its two arguments, if the associated Hessian is non negative definite. Accordingly, the integral of $h$ will be a convex functional of $f$. Hence, we only have to determine the conditions for the non negativity of the Hessian. 
By direct computation, we readily obtain that the Hessian matrix for the function $h(x, y)$ is

$$
H=\left[\begin{array}{cc}
\frac{\partial^{2} h}{\partial x^{2}} & \frac{\partial^{2} h}{\partial x \partial y} \\
\frac{\partial^{2} h}{\partial y \partial x} & \frac{\partial^{2} h}{\partial y^{2}}
\end{array}\right]=x^{\beta-2} y^{b-\beta-2}\left[\begin{array}{cc}
y^{2} \beta(\beta-1) & x y \beta(b-\beta) \\
x y \beta(b-\beta) & x^{2}(b-\beta)(b-\beta-1)
\end{array}\right] .
$$

The determinant of this matrix is

$$
D=-x^{2 \beta-2} y^{2(b-\beta)-2} \beta(b-\beta)(b-1),
$$

and its trace is

$$
T=x^{\beta-2} y^{b-\beta-2}\left(\beta y^{2}(\beta-1)+x^{2}(b-\beta)(b-\beta-1)\right) .
$$

Since we know that the determinant and trace of a matrix are respectively the product and sum of the eigenvalues, we see that the two eigenvalues are non negative if both the determinant and trace are non negative, for any $x$ and $y$. This entails here the conditions

$$
\begin{cases}\beta(b-\beta)(b-1) & \leq 0 \\ \beta(\beta-1) & \geq 0 \\ (b-\beta)(b-\beta-1) & \geq 0\end{cases}
$$

Actually, the first two conditions are sufficient since they imply the third one. With $\beta>0$, we finally obtain $\beta \geq b \geq 1$, which, given that $b=\beta(q-1)+1$, finally gives that $\phi_{\beta, q}[f]$ is a convex functional for

$$
2-\frac{1}{\beta} \geq q \geq 1
$$

Let us define by $\phi_{\beta, q}(m)$ and $I_{\beta, q}(m)$ the "thermodynamic" $(\beta, q)$-Fisher information considered as functions of an observable $m=E\left[|X|^{\alpha}\right]$ :

$$
\phi_{\beta, q}(m)=\inf _{f}\left\{\phi_{\beta, q}[f]: f \in \mathcal{P} \text { and } m=E\left[|X|^{\alpha}\right]\right\}
$$

The value of the information attached to the optimum distribution is denoted $\phi_{\beta, q}(m)$ - the use of the square brackets and parenthesis distinguishes between the functions of the state and the functions of the observable. Though the generalized Fisher information $\phi_{\beta, q}[f]$ is not a convex function of the 
probability density function for $q<1$, we should notice that the "thermodynamic" $(\beta, q)$-Fisher information $\phi_{\beta, q}(m)$ and $I_{\beta, q}(m)$ are in fact convex in $m$ for any $q>\max \{(n-1) / n, n /(n+\alpha)\}$. This is actually an immediate consequence of the Cramér-Rao inequalities (25) and (26). Indeed, any generalized $q$-Gaussian $G_{\gamma}$ with parameter $\gamma$ reaches the Cramér-Rao bound and we have, for instance for the first $(\beta, q)$-Fisher information:

$$
m_{\alpha}\left[G_{\gamma}\right]^{\frac{1}{\alpha}} \phi_{\beta, q}\left[G_{\gamma}\right]^{\frac{1}{\beta \lambda}}=m_{\alpha}[G]^{\frac{1}{\alpha}} \phi_{\beta, q}[G]^{\frac{1}{\beta \lambda}} .
$$

Choosing $\gamma$ such that $m=m_{\alpha}\left[G_{\gamma}\right]$, we obtain that the "thermodynamic" $(\beta, q)$-Fisher information $\phi_{\beta, q}(m)$ is

$$
\phi_{\beta, q}(m)=K m^{-\frac{\beta \lambda}{\alpha}},
$$

with $K=m_{\alpha}[G]^{\frac{\beta \lambda}{\alpha}} \phi_{\beta, q}[G]$. Hence, since $\alpha, \beta$ and $\lambda$ are positive, $\phi_{\beta, q}(m)$ is a convex function of $m$.

\subsection{Reciprocity relations and Legendre thermodynamics relationships}

In the formulation of standard thermodynamics, the Legendre structure is an important ingredient. We show here that the standard reciprocity relations and Legendre structure of thermodynamics are still valid for generalized Fisher information. The fact that the Legendre structure of thermodynamics is preserved for general maximum-entropy-like problems and is just a consequence of Jaynes' maximum entropy principle have already be shown in [59], and re-derived in the case of the Fisher information in [52, 56]. We extend this to the case of the generalized $(\beta, q)$-Fisher information. Actually, we provide here a simple and self-contained derivation of the fact that the thermodynamics-like Legendre structure is a general and direct consequence of a minimization principle.

We look for the more general distribution, in the Fisher sense, which is compatible with a prior information given as a mean value of some observable. This distribution is selected as the distributions with minimum $(\beta, q)$-Fisher information, and the related variational problem is stated as

$$
I_{\beta, q}(m)=\inf _{f}\left\{I_{\beta, q}[f]: f \in \mathcal{P}, E\left[A_{i}(X)\right]=m_{i}, i=1 . . M\right\},
$$

where $m=\left[m_{1}, m_{2}, \ldots, m_{M}\right]$ now denotes the vector of moments $m_{i}=$ $E\left[A_{i}(X)\right] i=1 \ldots M$. The problem consists in finding a distribution with minimum $(\beta, q)$-Fisher information on the set of all probability distributions with a series of fixed moments $m_{i}, i=1 \ldots M$. 
Let $\lambda$ be the vector of the Lagrange multipliers $\lambda_{i}$ associated with the $M+1$ constraints, including the constraint $E\left[A_{0}(x)\right]=E[1]=m_{0}=1$, and let us define the dual function $D(\lambda)$ by

$$
D(\lambda):=\inf _{f \geq 0}\left\{I_{\beta, q}[f]-\sum_{i=0}^{M} \lambda_{i}\left(\int_{\Omega} A_{i}(x) f(x) \mathrm{d} x-m_{i}\right)\right\} .
$$

It is well known that the maximum of the dual function is always less than or equal to the value of the primal problem: $\sup _{\lambda} D(\lambda) \leq I_{\beta, q}(m)$. Clearly, the dual function can be expressed in term of the function $I_{\beta, q}(\lambda)$ defined by

$$
I_{\beta, q}(\lambda):=\sup _{f \geq 0}\left\{\sum_{i=0}^{M} \lambda_{i} \int_{\Omega} A_{i}(x) f(x) \mathrm{d} x-I_{\beta, q}[f]\right\},
$$

and that gives

$$
D(\lambda)=\sum_{i=0}^{M} \lambda_{i} m_{i}-I_{\beta, q}(\lambda)
$$

If we denote by $\bar{A}$ the vector of moments $\bar{A}_{i}=\int_{X} A_{i}(x) f(x) \mathrm{d} x$, then among all distributions with the same moments $\bar{A}$, there exists a distribution with minimum $(\beta, q)$-Fisher information, say $I_{\beta, q}(\bar{A})$, and this distribution realizes the supremum in (70) for a given moment. Then it remains to explore all the possible moments $\bar{A}$ and select the one which maximizes the right hand side of (70).

In other words, if $S_{\bar{A}}$ is the set of distributions with given moments $S_{\bar{A}}=\left\{f \geq 0: \bar{A}_{i}=\int_{\Omega} A_{i}(x) f(x) \mathrm{d} x, i=0 \ldots M\right\}$, again with $A_{0}(x)=1$ and $m_{0}=1$, then

$$
\begin{aligned}
I_{\beta, q}(\lambda) & =\sup _{\bar{A}} \sup _{f \in S_{\bar{A}}}\left\{\sum_{i=0}^{M} \lambda_{i} \int_{\Omega} A_{i}(x) f(x) \mathrm{d} x-I_{\beta, q}[f]\right\}, \\
& =\sup _{\bar{A}}\left\{\sum_{i=0}^{M} \lambda_{i} \bar{A}_{i}-I_{\beta, q}(\bar{A})\right\}
\end{aligned}
$$

where $I_{\beta, q}(\bar{A})$ denotes the $(\beta, q)$-Fisher information of the distribution which realizes the supremum in (70) for a given moment. These simple relations directly imply the so-called reciprocity relations. Indeed, the maximum in (173) is attained for a value $\bar{A}_{\lambda}$ such that

$$
\nabla_{\bar{A}} I_{\beta, q}\left(\bar{A}_{\lambda}\right)=\lambda .
$$


On the other hand, (73) gives

$$
I_{\beta, q}(\lambda)=\sum_{i=0}^{M} \lambda_{i} \bar{A}_{i \lambda}-I_{\beta, q}\left(\bar{A}_{\lambda}\right) .
$$

Differentiating $I_{\beta, q}\left(\bar{A}_{\lambda}\right)$ with respect to $\lambda$ and taking into account (74), we obtain

$$
\nabla_{\lambda} I_{\beta, q}\left(\bar{A}_{\lambda}\right)=\left(\nabla_{\lambda} \otimes \bar{A}_{\lambda}\right) \cdot \nabla_{\bar{A}} I_{\beta, q}\left(\bar{A}_{\lambda}\right)=\left(\nabla_{\lambda} \otimes \bar{A}_{\lambda}\right) \cdot \lambda,
$$

where $x \otimes y$ denotes the outer product of the columns vectors $x$ and $y$, with $x \otimes y=x y^{t}$. The result (76) is nothing but a general form of the Euler theorem. Now, the differentiation of $I_{\beta, q}(\lambda)$ in (75) with respect to $\lambda$ gives

$$
\nabla_{\lambda} I_{\beta, q}(\lambda)=\overline{A_{\lambda}}+\left(\nabla_{\lambda} \otimes \overline{A_{\lambda}}\right) \cdot \lambda-\left(\nabla_{\lambda} \otimes \overline{A_{\lambda}}\right) \cdot \nabla_{\bar{A}} I_{\beta, q}\left(\bar{A}_{\lambda}\right)
$$

which, taking into account the Euler formula (76) yields the simple relation

$$
\nabla_{\lambda} I_{\beta, q}(\lambda)=\overline{A_{\lambda}}
$$

In fact, (73) expresses the fact that $I_{\beta, q}(\lambda)$ is the Legendre transform of $I_{\beta, q}(\bar{A})$, and relations (74) and (78) simply express that $\lambda$ and $\bar{A}$ are the dual variables linked by the induced Legendre structure. However, let us emphasize that $I_{\beta, q}(\bar{A})$ is not necessarily the Legendre transform of $I_{\beta, q}(\lambda)$; this will only be true if $I_{\beta, q}(\bar{A})$ is a convex function of $\bar{A}$.

Finally, observe that when we select the value of the Lagrange parameter $\lambda$ which maximizes the dual function, we obtain

$$
\nabla_{\lambda} I_{\beta, q}(\lambda)=m
$$

This gives the way to compute a value of $\lambda$, and therefore the associated probability distribution, such that the moment constraints $E\left[A_{i}\right]=m_{i}, i=$ $1 . . . M-1$ are satisfied.

\section{Conclusion}

In this paper, we have presented two extended forms of Fisher information that fit well in the context of nonextensive thermostatistics. Indeed, these $(\beta, q)$-Fisher information satisfy several extended properties that interrelate generalized $q$-Gaussians, generalized $(\beta, q)$-Fisher information and $q$-entropies. Among these properties, we have stressed that the generalized 
$q$-Gaussians are the solution of variational problems such as the minimization of the generalized $(\beta, q)$-Fisher information among distributions with a given $q$-entropy, or such as the minimization of the generalized $(\beta, q)$-Fisher information among distributions with a fixed moment. This complements the known fact that the generalized $q$-Gaussians maximize the $q$-entropies subject to a moment constraint. These results recover, as a particular case, known characterizations of the standard Gaussian. We have also introduced several information functionals minimized by the generalized $q$-Gaussian. In information theory, the important de Bruijn identity links the Fisher information and the derivative of the entropy. We have shown that this identity can be extended to generalized versions of entropy and Fisher information. More precisely, we have shown that for all distributions satisfying a nonlinear heat equation, then the generalized $(\beta, q)$-Fisher information naturally pop up in the expression of the derivative of the entropy. In a second step, we have examined further properties of the generalized Fisher information and of their minimization. In particular, we have considered the combination of two independent systems and shown that, though non additive, the generalized Fisher information of the combined system is upper bounded. In the case of mixing, we have shown that the generalized Fisher information is convex for $q \geq 1$. Finally, we have shown that the minimization of the generalized Fisher information subject to moment constraints leads to a Legendre structure analog to the Legendre structure of thermodynamics. We shall mention that though these different results were presented using an Euclidean norm on $\mathbb{R}^{n}$, they are actually also valid for arbitrary norms, following the general formulation in [30]. This means in particular that one can use a weighted norm such as $|x|=\sqrt{x^{t} C^{-1} x}$, where $C$ is a covariance matrix, which in turn leads to the characterization of elliptical generalized $q$-Gaussians. However, this does not directly account for matrix (or even higher order) constraints, and this still remains an open question in the present setting.

Further work should also examine whether a principle of minimum generalized $(\beta, q)$-Fisher information, together with the underlying Legendre structure, could lead to interesting predictions. Whatever the outcome, it is clear that the two generalized Fisher information appear as useful companions to the $q$-entropies, as demonstrated eg. by de Bruijn identity. The interplay between the extended $q$-entropies and generalized $(\beta, q)$-Fisher information could certainly be of value for the analysis and characterization of complex systems, e.g. following [23, 60, 61, 2]. 


\section{Appendix - Information measures of generalized q-Gaussians}

Let $g_{\gamma}(x)=\left(1-s \gamma|x|^{\alpha}\right)_{+}^{\frac{\nu}{s}}$. The corresponding generalized $q$-Gaussian is obtained as $G_{\gamma}(x)=g_{\gamma}(x) / \int g_{\gamma}(x) \mathrm{d} x$, with $\nu=1$ and $s=q-1$. In order to express the different information measures of generalized $q$-Gaussians, we use a closed-form expression given in the next proposition.

Proposition 6. Let $\alpha, p>0$, then consider the following quantity:

$$
\mu_{p, \nu}=\int|x|^{p}\left(1-s \gamma|x|^{\alpha}\right)_{+}^{\frac{\nu}{s}} d x
$$

We use the change of variable in polar coordinates $x=r u$, with $u=x /|x|$ and the representation of the Lebesgue measure $\mathrm{d} x=r^{n-1} \mathrm{~d} r \mathrm{~d} u$. In this expression $\mathrm{d} u$ denotes the surface element on the unit sphere and $\int d u=$ $n \omega_{n}$, where $\omega_{n}=\pi^{\frac{n}{2}} / \Gamma\left(\frac{n}{2}+1\right)$ is the volume of the $n$-dimensional unit ball. By the integral representations of the Beta function B, one gets the formula

$$
\begin{aligned}
\mu_{p, \nu}=\frac{1}{\alpha}(\gamma)^{-\frac{p+n}{\alpha}} n \omega_{n} \times \\
\begin{cases}(-s)^{-\frac{p+n}{\alpha}} B\left(\frac{p+n}{\alpha},-\frac{\nu}{s}-\frac{p+n}{\alpha}\right) & \text { for }-\frac{\nu \alpha}{(p+n)}<s<0 \\
s^{-\frac{p+n}{\alpha}} B\left(\frac{p+n}{\alpha}, \frac{\nu}{s}+1\right) & \text { for } s>0 \\
(\nu)^{-\frac{p+n}{\alpha}} \Gamma\left(\frac{p+n}{\alpha}\right) & \text { if } s=0\end{cases}
\end{aligned}
$$

From this general expression, we immediately identify the partition function of a generalized $q$-Gaussian $G_{\gamma}$ with parameter $\gamma: Z(\gamma)=\mu_{0,1}$, with $s=q-1$, which yields

$$
Z(\gamma)=\frac{1}{\alpha}(\gamma)^{-\frac{n}{\alpha}} n \omega_{n} \times \begin{cases}(1-q)^{-\frac{n}{\alpha}} B\left(\frac{n}{\alpha},-\frac{1}{q-1}-\frac{n}{\alpha}\right) & \text { for } 1-\frac{\alpha}{n}<q<1 \\ (q-1)^{-\frac{n}{\alpha}} B\left(\frac{n}{\alpha}, \frac{1}{q-1}+1\right) & \text { for } q>1 \\ \Gamma\left(\frac{n}{\alpha}\right) & \text { if } q=1 .\end{cases}
$$

Similarly, we obtain the information generating function

$$
\begin{aligned}
M_{q}\left[G_{\gamma}\right] & =\int G_{\gamma}(x)^{q} \mathrm{~d} x=\frac{\int g_{\gamma}(x)^{q} \mathrm{~d} x}{\left(\int g_{\gamma}(x) \mathrm{d} x\right)^{q}}=\frac{\mu_{0, q}}{\left(\mu_{0,1}\right)^{q}}, \\
& =\frac{\alpha q}{n(q-1)+\alpha q} \times \frac{1}{\left(\mu_{0,1}\right)^{q-1}}
\end{aligned}
$$

where the second line is obtained by simplification using the properties of the Beta functions, namely $B(x, y+1)=\frac{y}{x+y} B(x, y)$, and $B(x, y-1)=$ $\frac{x+y-1}{y-1} B(x, y)$. 
The information generating function, and thus the associated Rényi and Tsallis entropies are finite for $q>\max \{n /(n+\alpha), 1-\alpha / n\}$.

Likewise, the moment of order $p$ is given by

$$
m_{p}\left[G_{\gamma}\right]=\frac{\int|x|^{p} g_{\gamma}(x) \mathrm{d} x}{\int g_{\gamma}(x) \mathrm{d} x}=\frac{\mu_{p, 1}}{\mu_{0,1}}
$$

If $p=\alpha$, by the properties of the Beta functions, the expressions for the moment of order $p$ simplifies into

$$
m_{\alpha}\left[G_{\gamma}\right]=\frac{\mu_{\alpha, 1}}{\mu_{0,1}}=\frac{n}{\alpha} \frac{1}{\gamma(q-1)\left(\frac{1}{q-1}+\frac{n}{\alpha}+1\right)} \text { for } q>n /(n+\alpha)
$$

Let $b=\beta(q-1)+1$. The generalized $(\beta, q)$-Fisher information of the generalized Gaussian has the expression

$$
\phi_{\beta, q}\left[G_{\gamma}\right]=\frac{(\alpha \gamma)^{\beta}}{\left(\mu_{0,1}\right)^{b}} \int|x|^{\alpha}\left(1-(q-1)|x|^{\alpha}\right)_{+}^{\frac{b}{(q-1)}-\beta} \mathrm{d} x
$$

which, taking into account that $\beta(\alpha-1)=\alpha$, reduces easily to

$$
\phi_{\beta, q}\left[G_{\gamma}\right]=(\alpha \gamma)^{\beta} \frac{\mu_{\alpha, 1}}{\mu_{0,1}} \times \frac{1}{\left(\mu_{0,1}\right)^{\beta(q-1)}} .
$$

The second generalized $(\beta, q)$-Fisher information is given by

$$
I_{\beta, q}\left[G_{\gamma}\right]=\frac{\phi_{\beta, q}\left[G_{\gamma}\right]}{M_{q}\left[G_{\gamma}\right]^{\beta}}=(\alpha \gamma)^{\beta} \frac{\mu_{\alpha, 1}}{\mu_{0,1}} \times\left(\frac{\mu_{0,1}}{\mu_{0, q}}\right)^{\beta} .
$$

Let us finally evaluate the lower bound in the Cramér-Rao inequality (26). We have

$$
I_{\beta, q}\left[G_{\gamma}\right]^{\frac{1}{\beta}} m_{\alpha}\left[G_{\gamma}\right]^{\frac{1}{\alpha}}=\alpha \gamma\left(\frac{\mu_{0,1}}{\mu_{0, q}}\right)\left(\frac{\mu_{\alpha, 1}}{\mu_{0,1}}\right)^{\frac{1}{\beta}}\left(\frac{\mu_{\alpha, 1}}{\mu_{0,1}}\right)^{\frac{1}{\alpha}}=\alpha \gamma\left(\frac{\mu_{\alpha, 1}}{\mu_{0, q}}\right),
$$

since $\alpha^{-1}+\beta^{-1}=1$. By properties of the Beta functions, we obtain that the ratio simplifies to $\mu_{\alpha, 1} / \mu_{0, q}=n /(\gamma \alpha)$. Therefore, we get the equality $I_{\beta, q}\left[G_{\gamma}\right]^{\frac{1}{\beta}} m_{\alpha}\left[G_{\gamma}\right]^{\frac{1}{\alpha}}=I_{\beta, q}[G]^{\frac{1}{\beta}} m_{\alpha}[G]^{\frac{1}{\alpha}}=n$, which is the lower bound in (26) 


\section{References}

\section{References}

[1] J. S. Dehesa, P. Sánchez-Moreno, R. J. Yáñez, Cramér-Rao information plane of orthogonal hypergeometric polynomials, Journal of Computational and Applied Mathematics 186 (2) (2006) 523-541.

[2] J. S. Dehesa, S. López-Rosa, B. Olmos, R. J. Yáñez, Fisher information of d-dimensional hydrogenic systems in position and momentum spaces, Journal of Mathematical Physics 47 (5) (2006) 052104.

[3] J. S. Dehesa, A. Plastino, P. Sánchez-Moreno, C. Vignat, Generalized Cramér-Rao relations for non-relativistic quantum systems, Applied Mathematics Letters 25 (11) (2012) 1689-1694.

[4] T. M. Cover, J. A. Thomas, Elements of Information Theory, 2nd Edition, 2nd Edition, Wiley-Interscience, 2006.

[5] A. Dembo, T. Cover, J. Thomas, Information theoretic inequalities, IEEE Transactions on Information Theory 37 (6) (1991) 1501-1518.

[6] A. Stam, Some inequalities satisfied by the quantities of information of Fisher and Shannon, Information and Control 2 (2) (1959) 101-112.

[7] G. B. Folland, A. Sitaram, The uncertainty principle: A mathematical survey, Journal of Fourier Analysis and Applications 3 (3) (1997) 207238.

[8] K. D. Sen (Ed.), Statistical Complexity: Applications in Electronic Structure, 1st Edition, Springer, 2011.

[9] C. Tsallis, Introduction to Nonextensive Statistical Mechanics, first Edition, Springer, 2009.

[10] C. Beck, Generalised information and entropy measures in physics, Contemporary Physics 50 (4) (2009) 495.

[11] S. Golomb, The information generating function of a probability distribution, IEEE Transactions on Information Theory 12 (1) (1966) 75-77.

[12] E. Lutz, Anomalous diffusion and Tsallis statistics in an optical lattice, Physical Review A 67 (5) (2003) 051402. 
[13] V. Schwämmle, F. D. Nobre, C. Tsallis, q-Gaussians in the porousmedium equation: stability and time evolution, The European Physical Journal B-Condensed Matter and Complex Systems 66 (4) (2008) 537546 .

[14] C. Vignat, A. Plastino, Why is the detection of $q$-Gaussian behavior such a common occurrence?, Physica A 388 (5) (2009) 601-608.

[15] G. I. Barenblatt, On some unsteady motions of a liquid and a gas in a porous medium, Prikladnaja Matematika i Mechanika 16 (1952) 67-78.

[16] R. E. Pattle, Diffusion from an instantaneous point source with concentration dependent coefficient, Quart. J. Mech. Appl. Math. 12 (1959) 407-409.

[17] M. Del Pino, J. Dolbeault, Best constants for Gagliardo-Nirenberg inequalities and applications to nonlinear diffusions, Journal de Mathématiques Pures et Appliquées 81 (9) (2002) 847-875.

[18] M. Del Pino, J. Dolbeault, The optimal euclidean Lp-Sobolev logarithmic inequality, Journal of Functional Analysis 197 (1) (2003) 151-161.

[19] D. Cordero-Erausquin, B. Nazaret, C. Villani, A mass-transportation approach to sharp Sobolev and Gagliardo-Nirenberg inequalities, Advances in Mathematics 182 (2) (2004) 307-332.

[20] M. Agueh, Sharp Gagliardo-Nirenberg inequalities via p-Laplacian type equations, Nonlinear Differential Equations and Applications NoDEA 15 (2008) 457-472.

[21] L. Chimento, F. Pennini, A. Plastino, Naudts-like duality and the extreme Fisher information principle, Physical Review E 62 (5 Pt B) (2000) 7462-7465.

[22] M. Casas, L. Chimento, F. Pennini, A. Plastino, A. R. Plastino, Fisher information in a Tsallis non-extensive environment, Chaos,Solitons, \& Fractals 13 (3) (2002) 451-459.

[23] F. Pennini, A. R. Plastino, A. Plastino, Rényi entropies and Fisher informations as measures of nonextensivity in a Tsallis setting, Physica A 258 (3-4) (1998) 446-457.

[24] F. Pennini, A. Plastino, G. Ferri, Semiclassical information from deformed and escort information measures, Physica A 383 (2) (2007) 782796. 
[25] J. Naudts, Generalised exponential families and associated entropy functions, Entropy 10 (3) (2008) 131-149.

[26] J. Naudts, The q-exponential family in statistical physics, Central European Journal of Physics 7 (3) (2009) 405-413.

[27] S. Furuichi, On the maximum entropy principle and the minimization of the Fisher information in Tsallis statistics, Journal of Mathematical Physics 50 (1) (2009) 013303-12.

[28] S. Furuichi, On generalized Fisher informations and Cramér-Rao type inequalities, Journal of Physics: Conference Series 201 (2010) 012016.

[29] J.-F. Bercher, On generalized Cramér-Rao inequalities, generalized Fisher information and characterizations of generalized $q$-Gaussian distributions, Journal of Physics A: Mathematical and Theoretical 45 (25) (2012) 255303.

[30] J.-F. Bercher, On multidimensional generalized Cramér-Rao inequalities, uncertainty relations and characterizations of generalized $q$ Gaussian distributions, Journal of Physics A: Mathematical and Theoretical 46 (9) (2013) 095303.

[31] E. Lutwak, D. Yang, G. Zhang, Cramér-Rao and moment-entropy inequalities for Rényi entropy and generalized Fisher information, IEEE Transactions on Information Theory 51 (2) (2005) 473-478.

[32] E. Lutwak, S. Lv, D. Yang, G. Zhang, Extensions of Fisher information and Stam's inequality, IEEE Transactions on Information Theory 58 (3) (2012) $1319-1327$.

[33] J.-F. Bercher, On a $(\beta, q)$-generalized Fisher information and inequalities involving $q$-Gaussian distributions, Journal of Mathematical Physics 53 (6) (2012) 063303.

[34] O. Johnson, C. Vignat, Some results concerning maximum Rényi entropy distributions (2007) 339-351.

[35] L. Pardo, Order- $\alpha$ weighted information energy, Information sciences 40 (2) (1986) 155-164.

[36] O. Onicescu, Energie informationelle, Comptes Rendus de la Académie des Sciences Paris, Ser. A 263 (1966) 841. 
[37] A. Nachman, A. Callegari, A nonlinear singular boundary value problem in the theory of pseudoplastic fluids, SIAM Journal on Applied Mathematics 38 (2) (1980) 275-281.

[38] D. V. Widder, The Heat Equation, Academic Press, 1975.

[39] A. Ohara, Geometric study for the Legendre duality of generalized entropies and its application to the porous medium equation, The European Physical Journal B 70 (1) (2009) 15-28.

[40] J. L. Vázquez, The Porous Medium Equation: Mathematical Theory, 1st Edition, Oxford University Press, USA, 2006.

[41] J. L. Vázquez, Smoothing and Decay Estimates for Nonlinear Diffusion Equations: Equations of Porous Medium Type, Oxford University Press, USA, 2006.

[42] P. Lindqvist, Notes on the p-Laplace equation, Vol. 102, University of Jyväskylä, 2006.

[43] G. Savaré, G. Toscani, The concavity of Rènyi entropy power, arXiv:1208.1035.

[44] J. Naudts, Generalised Thermostatistics, 1st Edition, Springer, 2011.

[45] A. G. Bashkirov, Renyi entropy as a statistical entropy for complex systems, Theoretical and Mathematical Physics 149 (2) (2006) 15591573 .

[46] P. Jizba, T. Arimitsu, The world according to Renyi: thermodynamics of multifractal systems, Annals of Physics 312 (1) (2004) 17-59.

[47] E. Lenzi, R. Mendes, L. da Silva, Statistical mechanics based on Rényi entropy, Physica A: Statistical Mechanics and its Applications 280 (3-4) (2000) 337-345.

[48] A. Parvan, T. Biró, Rényi statistics in equilibrium statistical mechanics, Physics Letters A 374 (19-20) (2010) 1951-1957.

[49] T. Frank, A. Plastino, Generalized thermostatistics based on the Sharma-Mittal entropy and escort mean values, The European Physical Journal B - Condensed Matter and Complex Systems 30 (4) (2002) 543-549.

[50] C. Beck, E. G. D. Cohen, Superstatistics, Physica A 322 (2003) 267-275. 
[51] C. Tsallis, A. M. C. Souza, Constructing a statistical mechanics for Beck-Cohen superstatistics, Physical Review E 67 (2) (2003) 026106.

[52] B. R. Frieden, A. Plastino, A. R. Plastino, B. H. Soffer, Fisher-based thermodynamics: Its Legendre transform and concavity properties, Physical Review E 60 (1) (1999) 48-53.

[53] B. R. Frieden, P. M. Binder, Physics from Fisher information: A unification, American Journal of Physics 68 (11) (2000) 1064.

[54] B. R. Frieden, A. Plastino, A. R. Plastino, B. H. Soffer, Non-equilibrium thermodynamics and Fisher information: An illustrative example, Physics Letters A 304 (3-4) (2002) 73-78.

[55] B. R. Frieden, Science from Fisher Information: A Unification, Cambridge University Press, 2004.

[56] A. Plastino, A. Plastino, M. Casas, Fisher variational principle and thermodynamics, in: S. Sieniutycz, H. Farkas (Eds.), Variational and Extremum Principles in Macroscopic Systems, Elsevier, Oxford, 2005, pp. 379-394.

[57] M. Cohen, The Fisher information and convexity, IEEE Transactions on Information Theory 14 (4) (1968) 591-592.

[58] D. Boekee, An extension of the Fisher information measure, in: I. Csiszár, P. Elias (Eds.), Topics in Information Theory, Vol. 16, János Bolyai Mathematical Society and North-Holland, Keszthely, Hungary, 1977, pp. 113-123.

[59] A. Plastino, On the universality of thermodynamics' Legendre transform structure, Physics Letters A 226 (5) (1997) 257-263.

[60] C. Vignat, J. F. Bercher, Analysis of signals in the Fisher-Shannon information plane, Physics Letters A 312 (1-2) (2003) 27-33.

[61] E. Romera, J. S. Dehesa, The Fisher-Shannon information plane, an electron correlation tool, The Journal of Chemical Physics 120 (19) (2004) 8906-12. 